\title{
CHAOTIC FRACTAL MODELS GENERATED BY RECTANGULAR CELLS*
}

\author{
V. V. NOVIKOV ${ }^{1}$, K. W. WOJCIECHOWSKI ${ }^{2}$ AND V. P. PRIVALKO \\ ${ }^{1}$ Odessa National Polytechnical University, 1 Shevchenko Avenue, 65044 Odessa, Ukraine \\ e-mail: novikov@te.net.ua \\ ${ }^{2}$ Institute of Molecular Physics, Polish Academy of Sciences \\ M. Smoluchowskiego 17, 60-179 Poznań, Poland; e-mail: kww@man.poznan.pl \\ ${ }^{3}$ Institute of Macromolecular Chemistry, National Academy of Sciences of Ukraine \\ 263160 Kiev, Ukraine
}

(Rec. 18 December 2003)

\begin{abstract}
Properties of some chaotic fractal models constructed on hierarchies of rectangular cells (the latter being rectangular subsets of the square lattice) are investigated. Fractal dimensionalities and average neighbour numbers of structures generated by small rectangular cells $L_{x} \times L_{y} \quad\left(2 \leq L_{x} \leq 4\right.$, $\left.1 \leq L_{y} \leq 4\right)$ are derived. Generating probability functions and critical indices for the correlation length as well as for the percolation cluster density are calculated for the models considered. The calculations show that structures generated by anisotropic (rectangular) initial cells show much broader range of critical indices and other characteristic parameters than structures generated by 'isotropic' (square) initial cells.
\end{abstract}

\section{INTRODUCTION}

It is generally accepted that various physical systems can be thought of as fractals [1-6]. The examples are aggregates of particles in colloids, polymer molecules, percolating clusters, structures of some binary mixtures and polymers, etc. [1-16]. Thus, studies of model fractal structures can be helpful in modelling and understanding various properties of real systems. Such models can be also useful in constructing and testing various theoretical approximations. Exactly solvable models play an important role in this context as the results obtained for such models are free of, often uncontrolled, approximation errors.

Exactly solvable fractal structures can be obtained, e.g., on hierarchical lattices generated by finite subsets (further referred to as the generating cells) of simple periodic lattices. As an example, one can mention hierarchical lattices generated by finite square cells of a square lattice. Such systems have been considered in the literature, e.g. in [17] and [3], in the context of the percolation problem on the square lattice. Those classical studies, which concentrated on the 'isotropic' (square) cells of the square lattice, can be generalised to anisotropic (rectangular) generating cells. In the present paper we consider the percolation problem on hierarchical lattices generated by small, rectangular cells of the two-dimensional square lattice which are shown in Fig. 1. The aim of the study is to investigate the influence of the shape

\footnotetext{
* Dedicated to the memory of Professor Jacek Rychlewski
} 


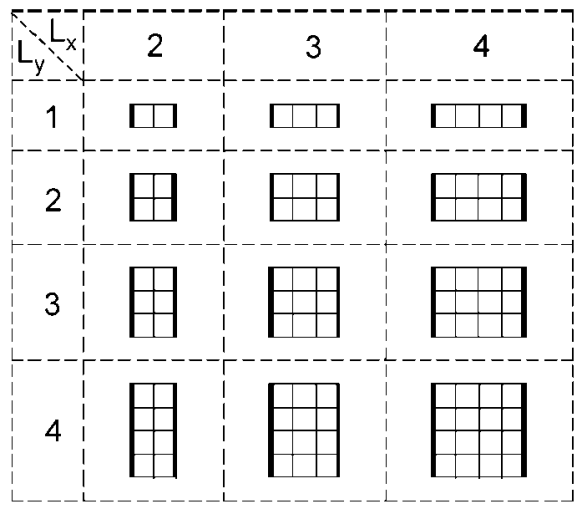

Fig. 1. The initial cells considered in this paper. Each bond marked by a thin line can be a connecting one with the probability $p$ or not connecting one with the probability $(1-p)$. The sides of the cells marked by thick lines are assumed to be always connecting

anisotropy of the generating cells on the average coordination number and critical properties of the model.

\section{FRACTALS GENERATED BY SQUARE CELLS}

Let us first consider fractal ensembles generated by square cells in which all bonds are identically coloured, i.e. the growth of the considered fractals is initiated on the twodimensional finite-size lattice $L_{0} \times L_{0}$. At the $n$-th stage, each bond of this lattice is replaced by a lattice generated at the preceding $(n-1)$-st stage. (The first step of the growth process for $L_{0}=2$ is illustrated in Fig. 2.) The linear scale of the system at the $\mathrm{n}$-th step is given by $L_{n}=L^{n}{ }_{0}$. The fractal dimensionality, $d_{f}^{0}\left(L_{0}\right)$, of the principal ensemble $\Omega_{0}\left(L_{0}\right)$ (with all bonds of the same colour) generated by the square cell $\left(L_{x}=L_{y}=L_{0}\right)$ can be determined from the relationship between its mass (i.e. the number of bonds which can be coloured; such bonds are represented by thin lines in Figs. 2, 3) and $L_{n}[18]$

$$
M_{n}\left(L_{0}\right)=L_{n}^{d_{f}^{0}\left(L_{0}\right)}
$$

Taking into consideration the validity of the following condition for square lattices:

$$
M_{n}\left(L_{0}\right)=\left(2 L_{0}^{2}\right)^{n}
$$

one obtains

$$
d_{f}^{0}\left(L_{0}\right)=2+\frac{\ln 2}{\ln L_{0}}
$$

As follows from Eq. (2), fractals of dimensionalities $2<d_{f}^{0}\left(L_{0}\right)<3$ can be obtained by changing the size $L_{0}$ of the generating (initial) square cell. For example, $d_{f}^{0}\left(L_{0}\right)=3$ for $L_{0}=2$, while $d_{f}^{0}\left(L_{0}\right) \rightarrow 2$ for $L_{0} \rightarrow \infty$. 
Fig. 2. The idea of building a self-similar lattice (on which the percolation is studied) by an iterative growth process of a square generating cell. At each stage of the growth process each thin bond of the generating cell is replaced by a structure obtained at the previous stage. The sides marked by thick lines are assumed to be always connecting
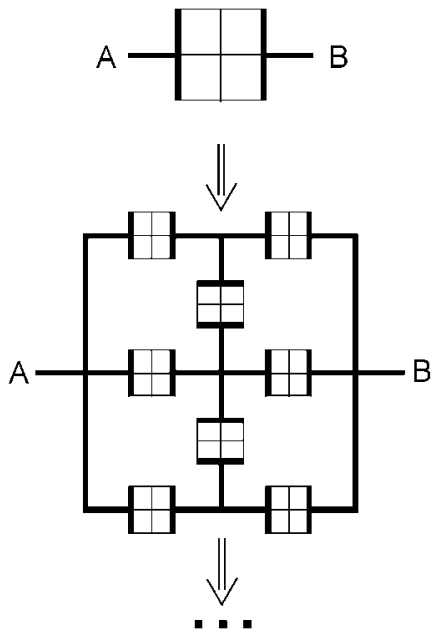

Consider now another ensemble of structures, $\Omega_{0}\left(L_{0}, p_{0}\right)$, which bonds can be coloured in black and white with $p_{0}$ as the probability for a bond to be black. In this case, one may distinguish between two basic states, in which the black bonds form either the bonded ensemble (BE) spanning the entire lattice space between two opposite faces, or the nonbonded ensemble (NBE) with no trajectory available to connect two opposite lattice faces.

The mass of the $\mathrm{BE}$ of black bonds in the vicinity of the critical point of transition $N B E \rightarrow$ BE depends on the linear scale $L_{n}$,

$$
M_{f}\left(L_{0}, p_{0}\right) \sim L_{n}^{d_{f}}\left(L_{0}, p_{0}\right)
$$

whereas its density may be defined as

$$
p_{B E}\left(L_{0}, p_{0}\right)=\frac{M_{f}\left(L_{0}, p_{0}\right)}{M_{n}\left(L_{0}\right)}=L_{n}^{d_{f}}\left(L_{0}, p_{0}\right)-d_{f}^{0}\left(L_{0}\right) .
$$

The correlation length $\xi$ of the $\mathrm{BE}$ is confined to the range of intermediate asymptotic, which may be defined as

$$
L_{0}<L<\xi \text {. }
$$

In this range, the BE is a fractal, i.e. it is geometrically similar to a percolating cluster, and its properties depend on the linear scale. Therefore, both the correlation length $\xi$ and the $P_{\mathrm{BE}}^{\infty}\left(L_{0}, p_{0}\right)$ of the $\mathrm{BE}$ (the upper index $\infty$ means that the limit $L_{n} \rightarrow \infty$ is taken) should scale with distance from the critical point (i.e., percolation threshold $p^{*}$ ) as

$$
\xi\left(L_{0}, p_{0}\right) \sim\left|p_{0}-p^{*}\right|^{-v\left(L_{0}\right)},
$$




$$
P_{B E}^{\infty}\left(L_{0}, p_{0}\right) \sim\left(p_{0}-p^{*}\right)^{\beta\left(L_{0}\right)}, \quad p_{0}>p^{*},
$$

where $p_{0} \rightarrow p^{*}+0^{+}$. Using (5) and (6) one can obtain the dependence of $P_{B E}^{\infty}\left(L_{0}, p_{0}\right)$ on the linear size of the system

$$
P_{B E}^{\infty}\left(L_{0}, p_{0}\right) \sim L_{n}^{-\frac{\beta\left(L_{0}\right)}{v\left(L_{0}\right)}}, \quad \text { when } \quad p_{0} \rightarrow p^{*}+0^{+} .
$$

Here, the critical indices for the BE correlation length and density are related through the fractal dimensionality $d_{f}\left(L_{0}\right)$ and $\beta\left(L_{0}\right), v\left(L_{0}\right)$ as

$$
\begin{gathered}
d_{f}\left(L_{0}\right)=d_{f}^{0}\left(L_{0}\right)-\beta\left(L_{0}\right) / v\left(L_{0}\right) \\
d_{f}\left(L_{0}\right)=\lim _{p_{0} \rightarrow p^{*+0^{+}}} d_{f}\left(L_{0}, p\right) \\
\beta\left(L_{0}\right)=\lim _{p_{0} \rightarrow p^{*}+0^{+}} \log _{10}\left[P_{B E}^{\infty}\left(L_{0}, p_{0}\right)\right] / \log _{10}\left[p_{0}-p^{*}\right] \\
v\left(L_{0}\right)=-\lim _{p_{0} \rightarrow p^{*}} \log _{10}\left[\xi\left(L_{0}, p_{0}\right)\right] / \log _{10}\left|p_{0}-p^{*}\right|
\end{gathered}
$$

An important characteristic of the chaotic fractal ensemble $\Omega_{0}\left(L_{0}, p_{0}\right)$ is the probability that a given configuration belongs to the $\mathrm{BE}$ (i.e., the percolation probability). At the 0 -th (i.e., initial) stage, this probability depends on the initial density of black bonds $p_{0}$ and on the size $L_{0}$ of the generating cell, and may be defined as the ratio of the number of bonding configurations to the total number of possible configurations. At the first growth step the length of the lattice rib is $L_{1}=L_{0}^{2}$, the density of black bonds is $p_{1}=R\left(L_{0}, p_{0}\right)$. In the next steps $L_{n}=L_{0} L_{n-1}$ and the probability of a bond to belong to the BE will depend on $p_{0}$, as follows,

$$
\begin{aligned}
& p_{2}=\left(L_{0}, p_{1}\right), \\
& \cdots \\
& p_{n}=R\left(L_{0}, p_{n-1}\right) .
\end{aligned}
$$

The unstable critical point $p^{*}=R\left(L_{0}, p^{*}\right)$ may be determined from the equality

$$
\lim _{n \rightarrow \infty} p_{n}=\left(\begin{array}{ll}
1, & p_{0}>p^{*} \\
0 & p_{0}<p^{*}
\end{array}\right.
$$

In any practical (finite precision) calculations the growth trajectory of the chaotic fractal ensemble $\Omega_{f}\left(L_{0}, p_{0}\right)$ ends at the $n$-th growth step (level) reaching a point indistinguishable from one of the fixed points 0 or 1 of the function $R\left(L_{0}, p\right)$ for the precision used.

The probability $P_{B E}^{(n)}\left(L_{0}, p_{0}\right)$ that a bond belongs to the BE at the $n$-th growth step, is calculated as 


$$
P_{B E}^{(n)}\left(L_{0}, p_{0}\right)=\prod_{i=0}^{n} R\left(L_{0}, p_{i}\right) .
$$

Equation (8) shows that a full (conducting) bond belongs to the "infinite" cluster only when it belongs to a cluster connecting the two opposite sides of the lattice on each iteration step $i-1, \ldots, n$. It follows from Eqs. (7) and (8) that

$$
\lim _{n \rightarrow \infty} P_{B E}^{(n)}\left(L_{0}, p_{0}\right)=\left\{\begin{array}{cc}
P_{B E}\left(L_{0}, p_{0}\right) & p_{0}>p^{*} \\
0 & p_{0}<p^{*}
\end{array} .\right.
$$

Thus, knowledge of the function $R\left(L_{0}, p\right)$ is crucial for determining the properties of the fractal model. For small initial lattices this function can be calculated exactly. The exact results for square generating cells of $L_{0}=2,3,4$, as well for more general case of rectangular generating cells, are given in the Appendix.

\section{FRACTALS GENERATED BY RECTANGULAR CELLS}

Consider now the fractal ensembles grown on rectangular subsets of the square lattice, $L_{x} \times L_{y}\left(L_{x} \neq L_{y}\right)$, further referred to as rectangular generating cells. In this case the characteristic length of the system, $L_{0}$, can be chosen in various ways. For $L_{x}>1$ (the case $L_{x}-1$ is not considered as a trivial one) the simplest and most natural choice is $L_{0}=L_{\mathrm{x}}$. As it can be seen in Fig. 3, for such a choice each iteration step increases the length of the system by the factor $L_{0}$. Thus, it is natural to choose $L_{n}=L^{n}$ as the characteristic length of the system at the $n$-th step.

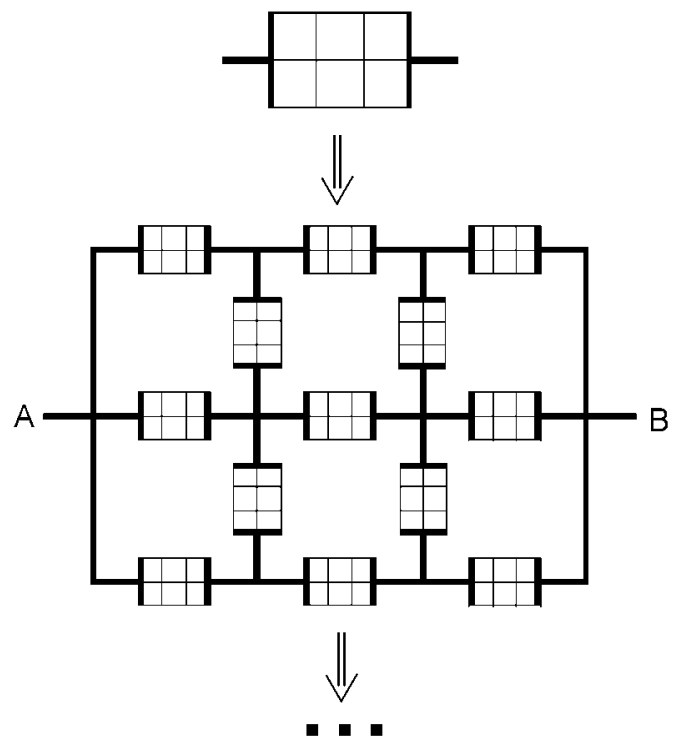

Fig. 3. The idea of building a self-similar lattice (on which the percolation is studied) by an iterative growth process of a rectangular generating cell; meaning of the thin and thick lines is as in the Fig. 2 
At the $n$-th growth step, the mass of the obtained 'fractal' ensemble will be

$$
M_{0}^{(n)}\left(L_{x} L_{y}\right)=\left(2 L_{x} L_{y}+L_{x}-L_{y}\right)^{n}
$$

so that using (1) one obtains the fractal dimensionality $d_{f}^{0}\left(L_{x}, L_{y}\right)$ of the system as

$$
d_{f}^{0}\left(L_{x}, L_{y}\right)=\frac{\ln \left(2 L_{x} \cdot L_{y}+L_{x}-L_{y}\right)}{\ln L_{x}} .
$$

One can easily check that $1<d_{f}^{0}\left(L_{x}, L_{y}\right)<\infty$, where the limits are reached for $L_{y} / L_{x} \rightarrow 0$ (this gives $\left.d_{f}^{0}\left(L_{x}, L_{v}\right)=1\right)$ and $L_{x} / L_{y} \rightarrow 0$ (this gives $d_{f}^{0}\left(L_{x}, L_{y}\right) \rightarrow \infty$

Probability functions $R\left(L_{x}, L_{y}, p\right)$ for fractal ensembles grown on several lattices (of the generating cells $L_{x} \times L_{y}$ where $2 \leq L_{x} \leq 4,1 \leq L_{y} \leq 4$ ) are presented in the Appendix, while calculated values of the percolation threshold $p^{*}$, fractal dimensionality of the ensemble at $p-1, d_{f}^{0}\left(L_{x}, L_{y}\right)$, mean fractal dimensionality at $p=p^{*},\left\langle d_{f}\right\rangle$, and critical indices $\beta\left(L_{x}, L_{y}\right)$ and $v\left(L_{x}, L_{y}\right)$ are listed in Table 1 . The index $\alpha_{1}$ in this table is calculated from the relation

$$
P_{B E}\left(L_{x}, L_{y}, p_{0}\right) \sim L_{n}^{-\alpha_{1}},
$$

i.e. $\alpha_{1}=\beta\left(L_{x}, L_{y}\right) / v\left(L_{x}, L_{y}\right)$. The results presented in Table 1 were obtained in the limit $p_{0} \rightarrow p^{*}$ and $\left\langle d_{f_{x}}\right\rangle=d_{f}^{0}\left(L_{x}, L_{y}\right)-\beta\left(L_{x}, L_{y}\right) / v\left(L_{x}, L_{y}\right)$.

Table 1. The percolation threshold, $p^{*}$, fractal dimensionality of the ensemble at $p=1, d_{f}^{0}\left(L_{x}, L_{y}\right)$, mean fractal dimensionality at $p=p,\left\langle d_{f x}>\right.$, and critical indices $\beta=\beta\left(L_{x}, L_{y}\right), \alpha_{1}$ and $v=v\left(L_{x}, L_{y}\right)$ for various initial rectangular cells. The index $\alpha_{1}$ is calculated from (12)

\begin{tabular}{cccccccc}
\hline$L_{x} \times L_{y}$ & $p^{*}$ & $d_{f}^{0}\left(L_{x}, L_{y}\right)$ & $\left\langle d_{f_{x}}\right\rangle$ & $\lambda_{p}=\left.\frac{d R}{d P}\right|_{p=p_{c}}$ & $v=\frac{\ln L_{x}}{\ln \lambda_{p}}$ & $\alpha_{1}$ & $\beta$ \\
\hline $2 \times 1$ & 0.500 & 2.322 & 1.322 & 1.625 & 1.428 & 1.000 & 1.428 \\
$2 \times 2$ & 0.304 & 3.000 & 1.282 & 1.823 & 1.154 & 1.718 & 1.983 \\
$2 \times 3$ & 0.223 & 3.459 & 1.293 & 1.912 & 1.069 & 2.166 & 2.315 \\
$2 \times 4$ & 0.178 & 3.807 & 1.314 & 1.962 & 1.028 & 2.493 & 2.564 \\
\hline $3 \times 1$ & 0.696 & 1.893 & 1.563 & 1.823 & 1.829 & 0.330 & 0.603 \\
$3 \times 2$ & 0.500 & 2.335 & 1.704 & 2.217 & 1.380 & 0.631 & 0.870 \\
$3 \times 3$ & 0.410 & 2.631 & 1.819 & 2.441 & 1.231 & 0.811 & 0.999 \\
$3 \times 4$ & 0.358 & 2.854 & 1.919 & 2.590 & 1.154 & 0.935 & 1.079 \\
\hline $4 \times 1$ & 0.777 & 1.730 & 1.548 & 1.911 & 2.139 & 0.182 & 0.389 \\
$4 \times 2$ & 0.590 & 2.085 & 1.704 & 2.441 & 1.553 & 0.381 & 0.591 \\
$4 \times 3$ & 0.500 & 2.322 & 1.822 & 2.766 & 1.363 & 0.500 & 0.681 \\
$4 \times 4$ & 0.451 & 2.500 & 1.926 & 3.097 & 1.226 & 0.574 & 0.704 \\
\hline
\end{tabular}

Closing these calculations, we should notice that, according to the construction procedure, see Fig. 3, the considered lattices are inhomogeneous, i.e. the coordination number, $Z$, for 
the investigated lattices depends on the lattice coordinates. Introducing the average coordination number

$$
<Z>=2(\text { total number of bonds }) /(\text { total number of sites })
$$

and taking into account that the considered lattices are self-similar, the average coordination number can be expressed by the length of the sides of the generating cell as

$$
\langle Z\rangle=2 \cdot \frac{2 L_{x} L_{y}+L_{x}-L_{y}-1}{L_{x} L_{y}+L_{x}-L_{y}-1} .
$$

It follows from (12) that for rectangular generating cells the average coordination number can vary in the range $3 \leq\langle Z\rangle \leq 6$. This range is by the factor $9 / 2$ broader than the range obtained for square generating cells $\left(L_{x}=L_{y}\right)$, for which $4 \leq<Z>\leq 14 / 3$.

Table 2. The average coordination number, $\langle Z\rangle$, and the average number of full bonds around a lattice vertex, $p^{*}\langle Z\rangle$ calculated for various initial rectangular cells at the percolation threshold, $p^{*}$

\begin{tabular}{ccc}
\hline$L_{x} \times L_{y}$ & $\langle Z>$ & $\left\langle Z>p^{*}\right.$ \\
\hline $2 \times 1$ & 4 & 2 \\
$2 \times 2$ & $14 / 3$ & 1.418 \\
$2 \times 3$ & 5 & 1.114 \\
$2 \times 4$ & $26 / 5$ & 0.924 \\
$3 \times 1$ & $7 / 2$ & 2.436 \\
$3 \times 2$ & 4 & 2 \\
$3 \times 3$ & $17 / 4$ & 1.743 \\
$3 \times 4$ & $22 / 5$ & 1.575 \\
$4 \times 1$ & $10 / 3$ & 2.591 \\
$4 \times 2$ & $34 / 9$ & 2.229 \\
$4 \times 3$ & 4 & 2 \\
$4 \times 4$ & $62 / 15$ & 1.864 \\
\hline
\end{tabular}

In Table 2 we present results obtained for the average coordination number $\langle Z\rangle$ and for the average number of full bonds around a lattice site at the percolation threshold (i.e. the product of $\langle Z\rangle$ and $p$ at the critical point) for the considered lattices. It can be seen there that for the inhomogeneous lattices considered this product can be much different from the value 2 which has been obtained for infinite uniform square lattice [3].

\section{SUMMARY AND CONCLUSIONS}

Chaotic fractal models generated by small rectangular cells were considered. Fractal dimensionalities of structures generated by various initial cells $(2 \times 1,2 \times 2,2 \times 3,2 \times 4$, $3 \times 1,3 \times 2,3 \times 3,3 \times 4,4 \times 1,4 \times 2,4 \times 3,4 \times 4)$ and their average neighbour numbers were calculated. Probability functions and critical indices for the correlation length and percolation cluster density were also derived. 
It has been shown that application of anisotropic (rectangular) initial cells in place of 'isotropic' (square) cells substantially increases the range of possible values of critical indices and other parameters characterising the models considered.

These results are of interest in the context of modelling of various properties of inhomogeneous media by the blob model discussed in [19-22]. In future works we plan to study effective conductivity and effective elasticity of fractal structures generated by various anisotropic cells. In particular, it is interesting if any auxetic [23] hierarchical structure (i.e. showing negative Poisson ratio, for a recent review see [24]) can be obtained on the basis of anisotropic generating cells.

\section{Acknowledgements}

We are grateful to Mr. M. Kowalik for help in prepararation of the figures and to Mr. P. K. Wojciechowski for independent determination of the generating functions $\mathrm{R}\left(L_{x}, L_{y}, p\right)$ collected in the Appendix. This work was supported in part by the (Polish) Committee for Scientific Research (KBN) grant 4T11F 01023, by the Polish Academy of Sciences, and by the Ukrainian National Academy of Sciences. Part of the calculations was performed at the Poznan Computer and Networking Centre (PCSS).

\section{References}

[1] B. B. Mandelbrot, Fractals: Form, Chance, andDimension (Freeman, San Francisco, 1977).

[2] P. de Gennes, Scaling Concepts in Polymer Physics (Cornell University Press, Ithaca, 1979).

[3] D. Stauffer and A. Aharony, Introduction to Percolation Theory, $2^{\text {nd }}$ edition (Talor \& Francis, London, 1992).

[4] M. Sahimi, Applications of Percolation Theory (Taylor \& Francis, London, 1994).

[5] V. P. Privalko and V. V. Novikov, The Science of Heterogeneous Polymers Structure and Thermophysical Properties (-Wiley, Chichester, 1995).

[6] P. Meakin, Fractals, Scaling and Growth far from Equilibrium, Cambridge Nonlinear Science Series, Vol 5 (Cambridge University Press, Cambridge, 1998)

[7] I. V. Zolotukhin, L. I. Yanchenko, and E. K. Belogonov, -ETP Letters 67, 720 (1998).

[8] S. S. Narine and A. G. Marangoni, Phys. Rev. E 59, 1908 (1999).

[9] T. H. Solomon, R. R. Hartley, and A. T. Lee, Phys. Rev. E 60, 3063 (1999).

[10] L. Cipelletti, S. Manley, R. C. Ball, and D. A. Weitz, Phys. Rev. Lett. 84, 2275 (2000).

[11] M. Lattuada, H. Wu, and M. Morbidelli, Phys. Rev. E 64, 61404 (2001).

[12] G. Reiter, G. Castelein, and --U. Sommer, Phys. Rev. Lett. 86, 5918 (2001).

[13] F. Yang and F. Pan, Phys. Rev. E 64, 51402 (2001).

[14] Z. W. Chen, X. P. Wang, S. Tan, S. Y. Zhang, -. G. Hou, and Z. Q. Wu, Phys. Rev. E 63, 65413 (2001).

[15] P. Cicuta and I. Hopkinson, Phys. Rev. E 65, 041404 (2002).

[16] F. Barra, B. Davidovitch, and I. Procaccia, Phys Rev E 65, 46144 (2002); see also references therein.

[17] P. -. Reynolds, H. E. Stanley, andW. Klein, Phys. Rev. B 21, 1223 (1980).

[18] V. V. Novikov and V. P. Belov, -. Exp. Theor. Physics 106, 780 (1994).

[19] V. V. Novikov, K. W. Wojciechowski, D. V. Belov, and V. P. Privalko, Phys. Rev. E 63, 36120 (2001).

[20] K. W. Wojciechowski and V. V. Novikov, TASK Quarterly 5, 5 (2001).

[21] V. V. Novikov and K. W. Wojciechowski, -. Exp. Theor. Physics 122, 462 (2002).

[22] V. V. Novikov and K. W. Wojciechowski, Physics of the Solid State 44, 2055 (2002).

[23] K. E. Evans, I.-. Hutchinson, and S.C. Rogers, Nature 353, 124 (1991).

[24] K. W. Wojciechowski, "Monte Carlo Simulations of model particles forming phases of negative Poisson ratio", in Properties and Applications of Nanocrystalline Alloys from Amorphous Precursors, edited by B. Idzikowski, P. Svec, and M. Miglierini (Kluwer Academic Publishers, Dordrecht, 2004) pp. 237-248; see also references therein. 


\section{APPENDIX}

The functions $R\left(L_{X}, L_{y}, p\right)$ derived for various generating cells are presented below.

Unit cell $2 \times 1$ :

$\mathrm{R}(1,2, p)=2 \mathrm{p}^{2}(1-p)^{3}+8 \mathrm{p}^{3}\left(1-p f+5 /(1-p)+p^{5} ;\right.$

Unit cell $2 \times 2$ :

$R(2, p)=\mathrm{R}(2,2, p)=3 /\left(1-p f+22 /(1-p)^{5}+56 \mathrm{p}^{4}(1-p)^{4}+54 /(1-p)^{3}+\right.$ $28 \mathrm{p}^{6}(1-p)^{2}+8 p^{1}(1-p)+p^{8}$

Unit cell $2 \times 3$ :

$\mathrm{R}(2,3, p)=4 /(1-\mathrm{p})^{9}+42 /(1-\mathrm{p})^{8}+178 /(1-p f+$ $382 p^{5}(1-p)^{6}+442 /(1-p)^{5}+328 /(1-p)^{4}+$

$165 /(1-p)^{3}+55 /(1-p)^{2}+11 /^{0}(1-p)+/^{1}$;

Unit cell $2 \times 4$ :

$\mathrm{R}\left(2,4, \_\mathrm{p}\right)=5 p^{2}(1-p)^{I 2}+68 \mathrm{p}^{3}(1-p)^{11}+398 /(1-p)^{10}+$

$1298 \mathrm{p}^{5}(1-\mathrm{p})^{9}+2575 /(1-p)^{8}+3288 /(1-p)^{1}+$ $2977 /(1-p)^{6}+2000 /(1-p)^{5}+1001 p^{10}(1-p)^{4}+$ $364 p^{11}(1-p)^{3}+91 /^{2}(1-p)^{2}+14 p^{13}\left(1-p+/^{4}\right.$;

Unit cell $3 \times 1$ :

$\mathrm{R}(3,1, p)=2 p^{3}(1-p)^{5}+14 /(1-p)^{4}+34 p^{5}(1-\mathrm{p})^{3}+25 /(1-p)^{2}+8 /(1-p)+p^{8} ;$

Unit cell $3 \times 2$ :

$\mathrm{R}(3,2, p)=3 /(1-p)^{10}+38 \mathrm{p}^{4}(1-\mathrm{p})^{9}+209 /(1-p)^{8}+$

$627 /(1-p)^{1}+1089 /(1-p)^{6}+1078 p^{8}(1-p)^{5}+$

$677 /\left(1-\mathrm{p}^{4}+283 /{ }^{\circ}(1-p)^{3}+78 \mathrm{p}^{11}(1-\mathrm{p})^{2}+13 /^{2}(1-p)+p^{13} ;\right.$

Unit cell $3 \times 3$ :

$$
\begin{aligned}
R(3, p)= & \mathrm{R}(3,3, p)=4 p^{3}(1-p)^{1}+72 /(1-p)^{14}+594 \mathrm{p}^{5}(1-p)^{13}+ \\
& 2936 /(1-\mathrm{p})^{12}+9582 /(1-\mathrm{p})^{11}+21470 /(1-\mathrm{p})^{10}+ \\
& 33494 /\left(1-p f+36774 \mathrm{p}^{10}(1-\mathrm{p})^{8}+29642 /{ }^{\wedge} 1-p f+\right. \\
& 18119 \mathrm{p}^{12}(1-p)^{6}+8514 \mathrm{p}^{13}(1-\mathrm{p})^{5}+3057 /^{4}(1-p)^{4}+ \\
& 816 \mathrm{p}^{15}(1-\mathrm{p})^{3}+153 /^{6}\left(1-p f+18 \mathrm{p}^{11}(1-p)+/^{8}\right.
\end{aligned}
$$

Unit cell $3 \times 4$ :

$$
\begin{aligned}
& \mathrm{R}\left(3,4, \_\mathrm{p}\right)=5 /(1-\mathrm{p})^{20}+116 \mathrm{p}^{4}(1-\mathrm{p})^{19}+1264 /(1-\mathrm{p})^{18}+ \\
& 8544 /\left(1-p f^{11}+39915 /(1-\mathrm{p})^{16}+135919 \mathrm{p}^{8}(1-\mathrm{p})^{15}+\right. \\
& 346869 /(1-\mathrm{p})^{14}+672995 /^{0}(1-\mathrm{p})^{13}+ \\
& 1001865 \mathrm{p}^{11}(1-\mathrm{p})^{12}+1158842 /^{2}(1-\mathrm{p})^{11}+ \\
& 1064880 /^{3}(1-\mathrm{p})^{10}+793300 /^{4}(1-p)^{9}+
\end{aligned}
$$




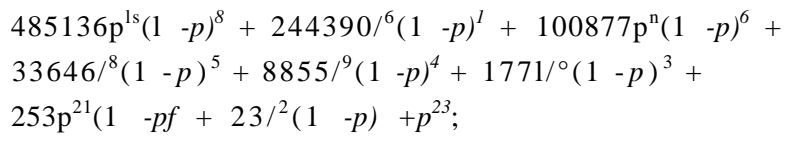

Unit cell $4 \times 1$ :

$5(4,1, \mathrm{p})=2 \mathrm{p}^{4}(1-p)+20 /(1-p)^{6}+80 /(1-p)^{5}+$ $152 /\left(1-\mathrm{p}^{4}+123 /(1-p)^{3}+51 /(1-p)^{2}+11 /{ }^{\circ}(1-\mathrm{p})+/^{1}\right.$;

Unit cell $4 \times 2$ :

$5(4,2, p)=3 /(1-p)^{14}+54 /(1-p)^{13}+445 /(1-p)^{12}+$ $2182 /(1-p)^{11}+6984 /(1-p)^{1 \circ}+15126 p^{9}(1-p)^{9}+$ $22288 \mathrm{p}^{1 \circ}(1-p)^{8}+22242 \mathrm{p}^{11}(1-\mathrm{p})^{1}+15628 \mathrm{p}^{12}(1-p)^{6}+$ $7974 /^{3}(1-p)^{5}+2988 /^{4}\left(1-\mathrm{p}^{4}+812 \mathrm{p}^{15}(1-p)^{3}+\right.$ $153 /^{6}(1-. p)^{2}+18 p^{11}(1-p)+/^{8} ;$

Unit cell $4 \times 3$ :

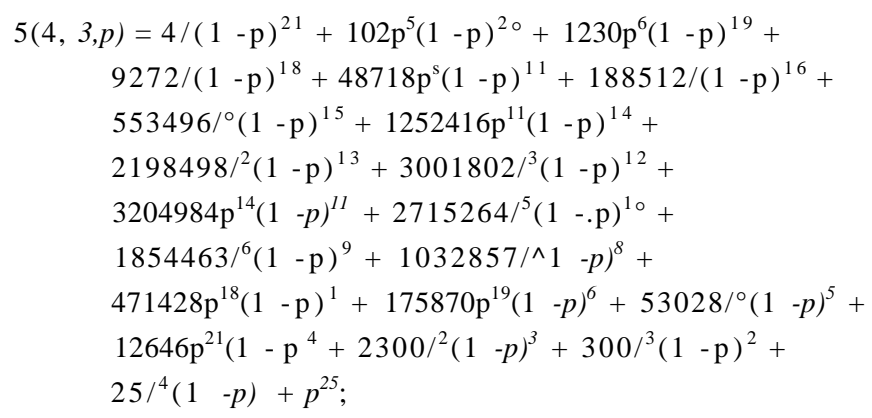

Unit cell $4 \times 4$ :

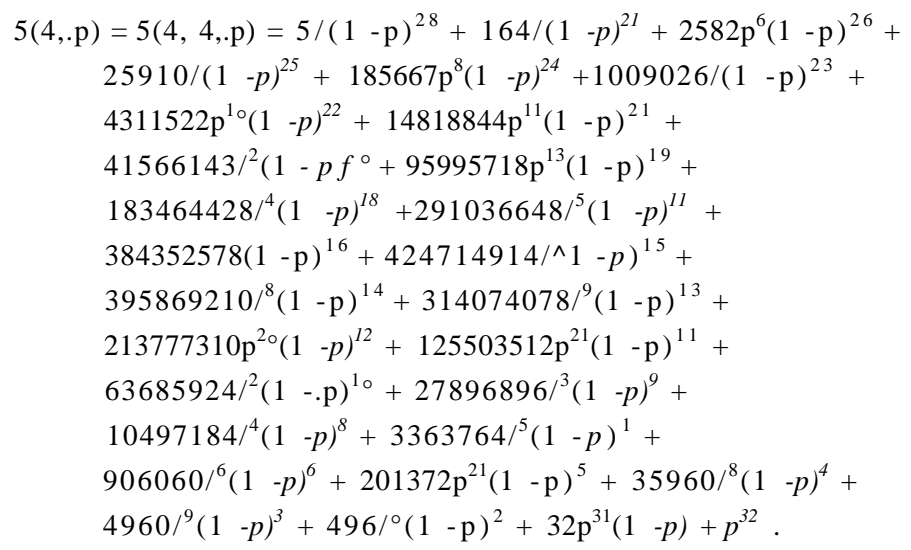

